\title{
Philosophiques
}

\section{Wittgenstein et les conditions d'une communauté linguistique}

\section{Denis Sauvé}

Volume 28, numéro 2, automne 2001

URI : https://id.erudit.org/iderudit/005679ar

DOI : https://doi.org/10.7202/005679ar

Aller au sommaire du numéro

Éditeur(s)

Société de philosophie du Québec

ISSN

0316-2923 (imprimé)

1492-1391 (numérique)

Découvrir la revue

Citer cet article

Sauvé, D. (2001). Wittgenstein et les conditions d'une communauté linguistique. Philosophiques, 28(2), 411-432. https://doi.org/10.7202/005679ar

\section{Résumé de l'article}

Pour certains interprètes des Recherches philosophiques, Wittgenstein souscrit à l'idée que l'emploi d'un langage est une institution sociale et que suivre une règle est nécessairement une pratique partagée ; d'autres estiment au contraire —à mon avis avec raison — qu'il admet la possibilité d'un langage parlé par un seul individu (à la condition qu'il ne soit pas " privé ") et des règles non communes. Je défends l'interprétation selon laquelle la question importante dans les Recherches n'est pas tellement de savoir si un idiolecte est possible (ou s'il peut y avoir des règles suivies par un seul individu) que de savoir ce qui découle, pour nos concepts (entre autres) de signification, de compréhension linguistique et de "suivre une règle ", du fait que la communication verbale soit - comme c'est normalement le cas - une pratique commune. 


\title{
Wittgenstein et les conditions d'une communauté linguistique
}

\author{
DENIS SAUVÉ \\ Cégep de Saint-Hyacinthe
}

\begin{abstract}
RÉSUMÉ. - Pour certains interprètes des Recherches philosophiques, Wittgenstein souscrit à l'idée que l'emploi d'un langage est une institution sociale et que suivre une règle est nécessairement une pratique partagée; d'autres estiment au contraire - à mon avis avec raison — qu'il admet la possibilité d'un langage parlé par un seul individu (à la condition qu'il ne soit pas « privé ») et des règles non communes. Je défends l'interprétation selon laquelle la question importante dans les Recherches n'est pas tellement de savoir si un idiolecte est possible (ou s'il peut y avoir des règles suivies par un seul individu) que de savoir ce qui découle, pour nos concepts (entre autres) de signification, de compréhension linguistique et de « suivre une règle», du fait que la communication verbale soit - comme c'est normalement le cas - une pratique commune.
\end{abstract}

\begin{abstract}
According to some interpreters of the Philosophical Investigations, Wittgenstein thinks that language-use is a social institution and that rule following is a shared practice. Others hold the opposite view and rightly so. They argue that Wittgenstein thinks there could be a language which is spoken by only one individual (provided it is not private) and unshared rules. In this paper I defend the following interpretation: The important question raised in the Investigations is not whether or not an idiolect is possible (or whether or not there could be unshared rules), it is rather what follows with respect to our concepts of meaning, understanding and rule-following from the fact that verbal communication is indeeed (normaly) a shared practice.
\end{abstract}

Wittgenstein fait dans le premier tiers de ses Recherches philosophiques (PU $\mathbb{S}$ 1-242) une série de remarques importantes sur la signification, la compréhension linguistique, l'explication du sens et le concept de "suivre une règle ». La difficulté de ces passages, comme de l'ensemble des Recherches, vient de ce qu'il n'indique pas toujours clairement les connexions entre ses différentes remarques et, surtout, qu'il dit peu de choses sur leur direction d'ensemble. Je défendrai l'interprétation suivante : une question à laquelle il tente de répondre dans ces passages - peut-être la question principale - est celle des conditions de possibilité d'une communauté linguistique, autrement dit la question : "Comment la communication verbale entre usagers d'une même langue est-elle possible ? "Si cette interprétation est correcte, une lecture du type de celle de Kripke (par exemple) ne nous donne pas une image exacte de ce que Wittgenstein se propose de faire dans les Recherches. La solution du "paradoxe sceptique " que l'on trouve selon lui dans les Recherches entraîne l'impossibilité d'un langage parlé par un seul individu : il n'y a pas de langage qui ne soit commun à plusieurs locuteurs ${ }^{1}$. (Hacker et Baker

1. Kripke, Saul, Wittgenstein on Rules and Private Language, Oxford, Blackwell, 1982, p. 109. 
ont soutenu au contraire qu'un langage que parlerait un seul individu est possible d'après Wittgenstein à la condition qu'un autre puisse le comprendre). Mon interprétation diffère de celle de Kripke au moins sur ce point : la question importante pour l'auteur des Recherches n'est pas tant de savoir si un langage est nécessairement partagé (une question dont je ne traiterai pas $\mathrm{ici}^{2}$ ) que de savoir ce qui découle, en ce qui concerne nos idées sur la signification et la compréhension, du fait que le langage que nous (les êtres humains) parlons soit un langage partagé ${ }^{3}$.

Ce qui suit se divise en quatre sections : (i) La première expose la réponse donnée dans les Recherches à la question : " En quoi la signification doit-elle consister pour que la communication soit possible entre utilisateurs d'une même langue ? " L'idée centrale des premiers paragraphes ( $P U \mathbb{S} \mathbb{S} 1$ à 25 ) est que la signification d'un mot est son « usage " ou son " rôle " dans des « jeux de langage ». (ii) Le critère de la compréhension d'un mot chez un locuteur est l'usage qu'il en fait : la façon dont il « opère » avec le mot est constitutif de sa compréhension. La deuxième section cite trois objections qu'adresse Wittgenstein au tenant du mentalisme qui estime que la compréhension est plutôt de l'ordre de choses « cachées » dans l'esprit des locuteurs. (iii) Les paragraphes 65 à 88 traitent du problème que posent quant à la possibilité d'une signification commune plusieurs expressions des langues naturelles n'ayant pas un usage fixe et clairement défini ou dont l'emploi n'est pas " partout circonscrit par des règles ". Le problème, dont il est question dans la troisième section, est celui de savoir comment une compréhension commune de ces expressions est possible et, en particulier, comment on peut en expliquer (ou communiquer) le sens. (iv) Enfin, la dernière section traite du " paradoxe » au sujet du concept de "suivre une règle » $(P U \mathbb{S} \mathbb{S} 185-242)$. $\mathrm{Si}$, comme le pense Wittgenstein, il existe toujours plus d'une façon de comprendre ou interpréter une règle d'usage (ce que je montre dans les deux précédentes sections), on peut se demander en quoi va consister son application correcte. C'est l'interlocuteur, dans les Recherches, qui soulève le problème mais, quoique Wittgenstein s'objecte à la formulation que ce dernier en

2. Voir cependant ci-dessous, note 20 .

3. Les abréviations pour les références aux écrits de Wittgenstein seront les suivantes : T pour Tractatus Logico-Philosophicus, Londres : Routledge and Kegan Paul, 1961 ; PU pour Philosophische Untersuchungen (Recherches philosophiques), éd. due aux soins de G. E. M. Anscombe et de R. Rhees, Francfort : Suhrkamp, 1969; BB pour The Blue and the Brown Books, Oxford : Blackwell, 2e éd., 1969 ; WLC pour Wittgenstein's Lectures, Cambridge 193235, éd. due aux soins de A. Ambrose, Chicago : University of Chicago, $1982 ; Z$ pour Zettel (Fiches), éd. due aux soins de G. E. M. Anscombe et de G. H. von Wright, Berkeley et Los Angeles : University of California Press, 1967 ; ÜG pour Über Gewissheit (On Certainty) éd. due aux soins de G. E. M. Anscombe et de R. Rhees, New-York : Harper and Row, 1969 ; BGM pour Bermerkungen über die Grundlagen der Mathematik, éd. due aux soins de G. E. M. Anscombe, de R. Rhees et de G. H. von Wright, Francfort : Suhrkamp, 1989; WLFM pour Wittgenstein's Lectures on the Foundations of Mathematics, Cambridge 1939, éd. due aux soins de C. Diamond, Chicago : University of Chicago Press, 1976. 
donne (parce que, dit-il, il commet un "malentendu»), il parle bien de "notre paradoxe ». Je présente dans cette section la « solution » qu'offre Wittgenstein à ce problème, une solution qui, je pense, présente une certaine similitude avec une "solution sceptique» au sens de Kripke ${ }^{4}$.

\section{La signification en tant qu'« usage dans le langage »}

Un passage des Recherches met en opposition deux approches de la communication :

[...] nous sommes tellement habitués à la communication par le langage dans une conversation qu'il nous semble que l'important consiste en ceci : un autre saisit le sens de mes mots - quelque chose de mental — il le prend pour ainsi dire dans son esprit. Si de plus il en fait ensuite quelque chose, cela n'appartient pas à la fonction immédiate du langage. $(P U \mathbb{S} 363)$.

Ou bien la communication consiste en une transmission de pensées véhiculées par les mots, ou bien "l'important"se trouve dans les activités des locuteurs, dans ce qu'ils "font avec les mot ». Le passage renvoie à l'opposition entre une conception plus ou moins traditionnelle de la communication, dont celle de Frege (à laquelle il fait peut-être allusion) serait un exemple, et celle que préconise Wittgenstein. On pourrait les décrire comme suit.

Il y a communication, pour Frege, quand l'auditeur a compris la signification des mots employés par le locuteur - lorsqu'il a saisi le sens, c'est-àdire la pensée qu'il veut lui transmettre ; la signification, comme dit ici Wittgenstein, est par essence "mentale ». Les pensées diffèrent cependant des « représentations " en ce qu'elles possèdent une sorte d' " objectivité » : les mêmes mots doivent exprimer pour tous les locuteurs du langage les mêmes pensées, ils doivent leur associer les mêmes sens ; les pensées demeureraient autrement incommunicables et privées. L'objectivité du sens est une condition d'un langage commun ${ }^{5}$.

Wittgenstein fait à plusieurs endroits dans ses écrits la critique des conceptions mentalistes de la signification et de la compréhension. Il est juste en un sens de dire que la communication consiste en une " transmission» de

4. On pourrait soutenir que la question du « langage privé » dans les Recherches (PU $\mathbb{S} \mathbb{S} 243-315)$ concerne les conditions d'un vocabulaire commun de mots de sensations. Il s'agit apparemment de savoir s'il peut y avoir des règles «privées » sous-jacentes à notre langage des sensations (des règles "privées " dans la mesure où chaque locuteur a une sorte d'accès privilégié à ses propres sensations). L' "argument du langage privé » est censé montrer l'impossibilité de règles de ce genre. J'ai défendu cette interprétation dans "L'argument du langage privé ", Dialogue, 24, 1985) et "Le problème du "langage privé " et la conception wittgensteinienne du langage ", Dialogue, 27, 1988).

5. Cf. Frege, G., "La pensée » dans Écrits logiques et philosophiques, trad. de C. Imbert, Paris, Seuil, 1971, pp. 180 et suiv. 
pensées, mais cela dépend de ce qu'on entend par le mot "pensée »- de ce que veulent dire les expressions « signifier» ou « comprendre quelque chose par un mot» (cf. sections 2 et 3 ). Wittgenstein rejette par ailleurs l'idée de l'objectivité (ou intersubjectivité) du sens comprise à la façon de Frege : il n'est pas nécessaire pour qu'on puisse légitimement parler du sens d'un mot (ou de l'identité ou non-identité du sens de deux mots) qu'il y ait quelque chose qui en soit le sens. Il refuse la "mythologie " (la réification) du sens.

En quoi alors réside l'objectivité de la signification ? La signification est objective si elle est l'" usage dans le langage ». Une condition d'un langage commun est que la signification soit l'" usage ".

Wittgenstein parle parfois, au lieu de l'« usage », du « rôle » ou de la "fonction » des mots dans des jeux de langage. C'est le rôle (la fonction) qu'il assume dans les activités des locuteurs dans des jeux de langage qui donne à un signe sa signification. On lit par exemple dans les Recherches : "l'expression " jeu de langage" doit mettre [...] en valeur le fait que parler un langage est une partie d'une activité ou d'une forme de vie. » (PU $\$ 23)$ Plus explicitement dans De la certitude : « Nos paroles [nos activités linguistiques] reçoivent leur sens de nos autres actions » (ÜG $\ 229$ ).

Un rapprochement entre le rôle des signes et l'institution de la monnaie peut expliquer en quel sens la signification est l'« usage dans le langage ». Supposons qu'un anthropologue veuille étudier les coutumes d'un groupe d'individus ayant trait à leurs activités d'échanges. Il ne pourrait pas identifier un certain type d'objet comme une pièce de monnaie (ou en général un instrument d'échange) s'il ne comprenait pas le sens des activités des membres du groupe quand ils l'emploient (dans des commerces, des banques, etc.). De même, un "interprète radical " (peut-être le même anthropologue) ne saurait pas ce que signifient leurs énoncés s'il ne comprennait pas le sens des autres activités qu'ils mènent dans des jeux de langage - ce qui n'est possible que s'il se réfère à la "façon commune d'agir des êtres humains ". (Wittgenstein écrit : " La façon commune d'agir des êtres humains est le système de référence au moyen duquel nous interprétons une langue étrangère " $[P U \$ 206])$. Or une supposition plausible est que le rôle d'une pièce de monnaie dans les activités des membres du groupe est constitutif du genre de chose en question : celle-ci n'est rien d'autre que le type d'objet - peu importe ses propriétés matérielles spécifiques - qui remplit ces fonctions dans leurs activités d'échange. On pourrait soutenir de façon analogue que leurs activités quand ils participent à des jeux de langage sont ce qui donne une signification à leurs énoncés (aux sons qu'ils émettent) ${ }^{6}$. Affirmer que la

6. Pour prendre un exemple, supposons que l'interprète veuille traduire des phrases dans lesquelles figurent deux types de signes (des énoncés de la forme " OX », " $O Y$ ", «OZ »,... et «PX », «PY », «PZ »,...). L'émetteur (A) est un « constructeur » (on constate qu'il est en train de construire un abri) et le récepteur (B) son aide (cf $P U \mathbb{S} 2)$. L'interprète 
signification est le rôle ou l'usage dans un jeu de langage revient ainsi à dire que l'observation des circonstances environnantes (des actions des participants, des objets qu'ils manipulent, regardent, comptent, etc.) permet de déterminer, du point de vue d'un interprète, la signification des signes et qu'elles sont en même temps constitutives de leur signification : il s'agit de critères de ce qu'ils signifient (au sens wittgensteinien du mot « critère $")^{7}$.

L'objectivité de la signification découle du caractère public de l'usage (et des autres activités) dans les jeux de langage. Wittgenstein soutient également que la compréhension linguistique est conceptuellement liée aux activités (entre autres aux actes de langage) régies par les règles du jeu : pour prendre son exemple de jeu de langage du début des Recherches ( $c f$. $P U \mathbb{2}$ 2), le constructeur sait ce que ses ordres signifient s'il les donne dans des circonstances "normales " (fixées par les règles du jeu) et son aide les comprend s'il les exécute correctement. La compréhension est l'aptitude à

observe que, lorsque A a épuisé le stock d'un certain type de matériau, A émet une phrase composée du signe " $\mathrm{O}$ » suivi du signe « $\mathrm{X}$ » et que, lorsque $\mathrm{B}$ l'entend, il va immédiatement chercher (disons) un certain nombre de briques (l'interprète voit que A avait épuisé son stock de briques). Quand la phrase est " $\mathrm{OY}$ » plutôt que «OX », il observe dans des circonstances similaires, que B va chercher des dalles au lieu de briques. Quand l'énoncé est " PX ", B va compter (plutôt que chercher) des briques là où elles sont entreposées, puis revient et indique le nombre de briques comptées en faisant un signe des doigts. Quand l'énoncé est « PY », il agit de même après être allé compter des dalles. Et ainsi de suite. Si en s'appuyant sur la «façon d'agir commune des êtres humains " l'interprète peut comprendre les activités du constructeur et de son aide (ériger un mur de briques, aller chercher des dalles, compter des briques, etc.), il peut dire quelles fonctions (quels rôles) ont les signes et il est capable de les traduire. La phrase " $\mathrm{OX}$ » se traduit par "Va chercher des briques " ( $\mathrm{O}$ » signifie "Va chercher... et « $\mathrm{X}$ » signifie «briques"), parce que (au sens où on cite un critère) lorsque que " $\mathrm{OX}$ » est prononcée, A est à court de briques et $\mathrm{B}$ (lorsqu'il l'entend) va chercher des briques (l'interprète observe que A aurait besoin de briques pour construire ce mur, etc.) ; et, également, parce que, lorsque la phrase est " $\mathrm{OY}$ ", A n'a plus de dalles et B (lorsqu'il l'entend) va chercher des dalles et parce que, lorsque « PX » est prononcée, B va compter les briques, puis revient vers A et lui signale le nombre de briques, etc. L'ensemble de ces faits, sur lesquels se base l'interprète pour traduire " OX », est constitutif de la signification de la phrase.

7. Une conception de la signification proche de celle de Wittgenstein, je pense, est la conception de D. Føllesdal qui écrit : " [...] mon point de vue est celui très traditionnel et classique [d'après lequel] le langage est établi, appris et utilisé dans un contexte public où nous sommes entourés d'objets et d'événements publiquement observables et d'autres personnes qui perçoivent et manipulent ces mêmes objets et événements et que nous percevons en train de les percevoir et manipuler. Là où je vais au-delà de la tradition, c'est lorsque je soutiens qu'il n'y a rien d'autre dans la signification que ce qui résulte de cette interaction publique. » ("In What Sense is Language Public ? ", dans Leonardi, P. et Santambrogio, M., dirs., On Quine. New Essays, Cambridge, Cambridge University Press, 1995. p. 54). Il formule ailleurs comme suit ce point de vue : "La signification d'une expression [appartenant à un langage] est le produit conjoint [joint product] de toutes les données [evidence] qui aident ceux qui font l'apprentissage du langage et ceux qui en font usage à déterminer cette signification ". ( Indeterminacy and Mental States ", dans Barrett, R.B. et Gibson, R.F., dirs., Perspectives on Quine, Cambridge, MA, Blackwell, 1990. p. 103). 
" opérer avec les mots ${ }^{8}$ (cf.PU $\$ 6:$ "Ne pouvons-nous dire de celui qui agit de telle ou telle façon en réponse à l'ordre ["Apporte une dalle !»] qu'il le comprend ? » $)^{9}$.

\section{Mentalisme et signification}

On pourrait objecter que la communication doit consister en une " transmission de pensées » - c'était la première approche dans le passage cité au début - quand par exemple la situation est la suivante : A regarde par la fenêtre, il voit qu'il pleut et prononce les mots «Il pleut dehors » en s'adressant à B qui s'apprêtait à sortir ; B, qui l'entend, prend un parapluie et sort. Il semble qu'on puisse décrire ce qui s'est passé en disant que A a formé la pensée qu'il pleut dehors et l'a exprimée par la phrase correspondante, ce qui a induit chez B la même pensée (et explique en partie son comportement). L'objection peut prendre deux formes : (a) la pensée du locuteur, ce qu'il signifie par les mots "Il pleut dehors ", et celle de son auditeur qui les comprend sont des épisodes de leur vie mentale (ou peut-être des " objets » de pensée internes, des « jugements ", etc.) — c'est l'objection que fait le mentaliste; (b) ou bien ce sont des états ou processus de leur système nerveux ou des états hypothétiques dans un modèle de l'esprit grâce auxquels on peut prédire et expliquer causalement leurs comportements ( $c f . B B$ p. 6). La réponse de Wittgenstein à la seconde forme de l'objection revient essentiellement à dire que les concepts de signification et de compréhension figurant dans nos explications courantes diffèrent de ceux d'une psychologie scientifique ou des sciences du cerveau : les critères usuels de la compréhension ont trait aux comportements des locuteurs et non à des processus dans leur système nerveux ou leur " appareil psychique " (cf.PU $\mathbb{S} 149)$. Je ne développerai pas cette réponse ${ }^{10}$. Je citerai en revanche trois arguments qu'il oppose au mentaliste.

8. L'expression « opérer avec des mots " («mit Worten operieren») apparaît dans le premier paragraphe des Recherches. Après avoir décrit un jeu de langage, Wittgenstein fait remarquer : "C'est ainsi et d'autres façons semblables que l'on opère avec des mots. » Il est clair qu' " opérer avec des mots" signifie la même chose que "faire usage de mots " au sens où il emploie cette dernière expression. Pour faire un parallèle avec le jeu d'échecs : de même qu' " opérer avec » un pion n'est pas seulement " manipuler » une pièce de bois en la déplaçant sur un échiquier mais " jouer » avec la pièce (en faire usage) en accord avec des règles, " opérer avec des mots » consiste non simplement à émettre des sons (ou inscrire des signes) mais à les employer dans le contexte de jeux de langage (décrire un jeu de langage est donc toujours en même temps décrire un usage ou une certaine façon d'opérer avec les mots).

9. J'ai développé les idées de Wittgenstein sur la signification dans « La seconde théorie du langage de Wittgenstein ", Philosophiques, 22, 1995, "Interprétation, signification et “ usage " chez Wittgenstein ", Dialogue, 35, 1996 et "La philosophie du langage de Wittgenstein selon Michael Dummett ", Dialogue, 37, 1998.

10. Voir cependant ci-dessous note 22. 
a) A et $\mathrm{B}$ pourraient avoir la même chose " à l'esprit " lorsque A prononce et B entend les mots «Il pleut dehors » mais il se peut que B les comprenne ou les interprète d'une façon différente de la façon dont A les comprend. Wittgenstein invoque l'exemple un peu différent de quelqu'un qui donne une définition ostensive d'un mot. A veut expliquer à $\mathrm{B}$ la signification de « cercle » et lui dit " On appelle cela un cercle » en montrant un objet circulaire. Or une définition ostensive, dit-il, " peut être variablement interprétée dans tous les cas » $(P U \mathbb{S} 28)$. Mais le tenant du mentalisme pourrait faire remarquer qu'il suffit que $\mathrm{B}$ ait la même "expérience " (Erlebnis) que A lorsqu'il lui donne la définition ou qu'il « devine » ce que A a devant l'esprit pour qu'il comprenne et fasse le même usage du mot " cercle » : si son interprétation diffère de celle de A, c'est qu'il n'a pas correctement " deviné » ce qu'il «voulait dire ». Mais admettons que, au moment où il donnait la définition, A concentrait son attention sur un objet circulaire et suivait son contour du regard $(c f . P U \mathbb{S} 34)$ : n'est-il pas possible que B, qui l'observait, ait fait la même " expérience » ou ait ressenti ( «dans son esprit ») la même chose que A mais interprète autrement la définition ? Il semble que ce soit en effet possible. En quoi dès lors consiste le fait de "comprendre" ( "signifier») ou "interpréter" (d'une façon particulière) la définition ? Il écrit : « [...] ni l'expression " comprendre [meinen] de telle ou telle façon la définition " [chez A] ni " l'interpréter [deuten] de telle ou telle façon " [chez $B]$ ne désigne un processus [dans leurs esprits] qui accompagne l'action de prononcer et d'entendre la définition » $(P U \mathbb{S} 34)$. Si la compréhension (ou l'interprétation) n'est pas un tel processus, en quoi consiste-t-elle ? La compréhension peut entre autres « consister dans la façon dont [B] fait usage du mot qui a été défini, dans ce qu'il montre par exemple quand on lui

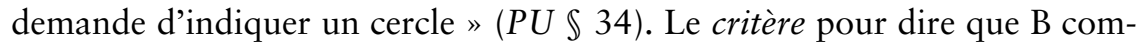
prend correctement la définition ou pour dire qu'il lui donne la même interprétation ou le critère pour dire qu'il y a eu communication entre $\mathrm{A}$ et $\mathrm{B}-$ ce sont les circonstances (publiques et intersubjectives) dans lesquelles les mots ont été employés ( $c f$. par exemple $P U \mathbb{1 5 4}$ ), non un processus de leurs esprits, donc les activités consistant à montrer un cercle, à aller chercher un parapluie, etc. - bref la façon dont ils opèrent avec les mots dans les jeux de langage ( $c f$. aussi $B B$ p. 2).

b) Une seconde objection est qu'il n'est pas nécessaire pour qu'il y ait compréhension que quelque chose se passe dans leurs esprits ( $c f . B B$ p. 3). B peut comprendre ce que A veut lui faire savoir sans que quoi que ce soit lui vienne à l'esprit - ce qui bien sûr n'empêche pas de leur attribuer la pensée qu'il pleut. Mais ici encore le critère pour dire que $B$ a eu cette pensée (comme pour dire qu'il a compris l'énoncé de A) réside dans les circonstances du jeu de langage, non en un occurrence mentale (par exemple dans l'action de dire dans son for intérieur "Ah! Il pleut dehors » - même si quelque chose de ce genre a pu se produire). 
c) Enfin la dernière objection est qu'il n'est pas suffisant qu'ils aient eu une « pensée » (toujours au sens d'un état ou processus interne) pour que $\mathrm{A}$ ait signifié quelque chose par ses mots ou que B l'ait compris. Le mentaliste pourrait en effet répondre que cela est parfois nécessaire. Pour montrer que ce n'est pas suffisant, Wittgenstein fait d'abord remarquer dans le Cabier bleu que, pour éliminer le caractère en apparence mystérieux des phénomènes mentaux (pour le mentaliste, seuls des phénomènes mentaux " cachés » peuvent rendre compte des phénomènes linguistiques "visibles " ou « donner vie » à des signes en eux-mêmes « inertes ${ }^{11}$ ), on peut adopter la "méthode " qui consiste à les remplacer par les processus " externes " (publiquement accessibles) correspondants (BB p. 13). Admettons avec le mentaliste qu'il soit dans certains cas nécessaire quand on entend le mot " rouge » que l'on ait une image mentale de la couleur rouge pour savoir ce que le mot signifie. La «méthode " revient à substituer au processus dans l'imagination l'action d'observer un objet tel un échantillon de la couleur rouge ( « Nous pourrions très bien pour nos propres fins remplacer tout processus d'imagination par le processus consistant à regarder un objet ou bien à peindre, dessiner ou façonner un modèle ; et tout processus consistant à se parler à soi-même par [l'action] de parler à haute voix ou d'écrire » $[B B \mathrm{p}$. 4]). Wittgenstein pose la question : "Pourquoi le signe écrit [le mot " rouge"] auquel on aurait joint cette image peinte [de la couleur rouge] devrait-il être vivant si le signe écrit seul était mort ? » (BB p. 5) Si ce n'est pas l'image peinte de la couleur rouge jointe au mot qui peut lui conférer une signification ou si elle ne suffit pas à le faire, pourquoi l'image dans l'imagination suffirait-elle?

L'objection devient plus explicite dans les Recherches (PU $\mathbb{S} \mathbb{S} 139$ 141). Supposons que, pour ordonner à $B$ d'aller chercher un objet de forme cubique, A prononce le mot " cube » et qu'il vienne à l'esprit de B l'image d'un cube (B n'a compris l'ordre, estime le mentaliste, qu'à la condition que l'image lui soit venue à l'esprit). Wittgenstein fait remarquer comme dans le Cahier bleu : «[...] n'est-il pas évident qu'il n'est pas du tout essentiel que cette image soit dans son imagination et non pas plutôt devant lui comme un dessin ou un modèle ou encore comme un modèle qu'il a construit ? "Et il pose cette question : " [e]n quel sens l'image peut-elle s'accorder ou ne pas s'accorder avec l'usage du mot " cube ” ? ( $P U \mathbb{\$} 139)$ Il semble qu'elle ne s'accorderait pas si B allait chercher un prisme triangulaire au lieu d'un cube. Or, dit-il, « il est facile de se représenter une méthode de projection suivant

11. Dans le même passage du Cahier bleu (p. 4), Wittgenstein décrit comme suit le point de vue de Frege : "Sans le sens, ou sans la pensée, une proposition est une chose totalement inerte et dénuée d'intérêt. Il semble clair en outre qu'aucune addition de signes inorganiques ne peut rendre vivante la proposition. Et la conclusion qu'on tire de cela est que ce qui doit être ajouté aux signes inertes pour rendre vivante la proposition est quelque chose d'immatériel doué de propriétés différentes de celles des simples signes ». 
laquelle l'image s'accorde après tout [avec l'usage consistant à aller chercher un prisme triangulaire] » (Ibid.). Certes l'image suggère un certain usage (aller chercher un cube) - celui qu'on qualifierait probablement de " normal " - mais on pourrait en imaginer un autre (aller chercher un prisme triangulaire) si on faisait varier la méthode de projection. On serait peut-être tenté de dire que, si on adjoignait à l'image un "schéma » (disons une image représentant deux cubes reliés par des lignes de projection), il n'y aurait plus dans ce cas qu'une façon de l'« appliquer " (puisqu'on aurait alors fixé la méthode de projection). Une supposition qui ne nous mène pas plus loin, répond Wittgenstein, car nous pouvons aussi imaginer "différentes applications de ce schéma » $(P U \mathbb{S} 141)$ : si on peut interpréter l'image en lui associant telle ou telle méthode de projection, il peut également y avoir différentes interprétations de cette méthode (différentes façons d'appliquer le schéma). Est-ce que cela entraîne que n'importe quelle application pourra s'accorder avec l'image et qu'il ne pourra jamais y avoir "collision », comme dit Wittgenstein, entre celle-ci et son application ? Il peut au contraire y avoir collision, comme il le fait ensuite remarquer, " dans la mesure où l'image nous fait nous attendre à un autre usage [aller chercher un cube et non un prisme triangulaire], puisque les gens font en général de cette image cette application " (Ibid.) - une remarque qu'il fait en passant mais qui s'avère plus loin importante ( $c f$. section 4$)$.

Je citerai un dernier passage qui présente également une critique du mentalisme et anticipe en même temps des développements importants des Recherches auxquels je reviens plus loin. Le mentaliste pourrait répliquer que, loin d'être constitutif de la compréhension, l'usage fait des mots n'en est qu'une "application » ou une manifestation. Wittgenstein fait dire ici au mentaliste : " [l'usage] est une application de la compréhension. La compréhension elle-même est un état duquel surgit l'usage correct " (PU $\mathbb{S} 146)$. L'exemple qu'il utilise est celui d'une situation dans laquelle A enseigne à $\mathrm{B}$ comment développer des suites de nombres : B a compris ce qu'on veut lui enseigner s'il est lui-même capable de poursuivre une série de nombres (c'est là notre critère pour dire s'il a ou non compris). Or on peut se demander à quoi pense le mentaliste lorsqu'il affirme que poursuivre la série n'est qu'une " manifestation » de l'« état » de compréhension chez l'apprenti. Il semble, répond Wittgenstein, qu'il pense à " la dérivation d'une série à partir d'une formule algébrique » ou à quelque chose d'" analogue » $(P U \mathbb{S} 146)$. Mais, comme il le fait ensuite remarquer, "nous sommes déjà passés par là » (sans doute une allusion au $\$ 141$ cité ci-dessus) : « Nous pouvons justement penser à plus d'une application d'une expression algébrique et chaque type d'application peut à son tour être formulé algébriquement ; mais bien entendu cela ne nous mène pas plus loin ». Il ajoute : "L'application demeure un critère de la compréhension » (ibid.).

On peut relever trois points au sujet de ce passage : (i) Wittgenstein fait à nouveau appel à la "méthode » du Cahier bleu. La compréhension appa- 
raît comme distincte de l'usage ; elle est l' "état " supposé être à la source de l'usage. Mais quel genre d'état est-ce et en quel sens dire, comme le mentaliste, que l'usage «surgit » de la compréhension ? L'état, répond Wittgenstein, ne peut être que quelque chose du genre d'une formule algébrique (ou quelque chose d' "analogue ") et, pour expliquer comment peut en " surgir " un usage, on n'a pas d'autre modèle que la dérivation d'une série à partir d'une formule algébrique (comme dans l'exemple précédent on n'avait pas d'autre modèle que la « dérivation » d'une action à partir du dessin d'un cube). (ii) D'autre part, de même qu'il est possible d'interpréter ou projeter de plus d'une façon le dessin ou l'image d'un cube (une définition

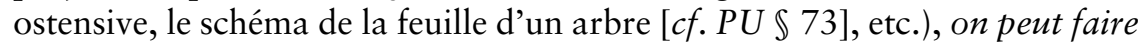
toutes sortes d'applications d'une formule algébrique et, comme on peut variablement interpréter l'image d'un cube accompagnée d'une méthode de projection, on peut imaginer différentes applications de l'expression algébrique de chacune des applications de la formule initiale (ce qui vaut également pour chacune de ces expressions) et il semble que ce soit vrai en général de toute règle ( $c f$. ci-dessous, sections 3 et 4). (iii) Enfin, à l'affirmation voulant que le critère pour dire que l'apprenti comprend (et ce qui est en même temps constitutif de sa compréhension) soit l'existence d'un « état » de son esprit (ou peut-être de son cerveau), lequel serait à la source de l'usage, la réponse est que c'est l'application (ici l'application de la formule algébrique, c'est-àdire l'usage dans le cas de la compréhension linguistique) qu'il faut considérer plutôt comme le critère de sa compréhension.

Il est important, je pense, de souligner que Wittgenstein ne souscrit pas à une forme de béhaviorisme suivant laquelle la signification et la compréhension se réduisent en fin de compte à des " comportements " : il est clair d'un côté que le point de vue adopté dans la description des actions est celui de la psychologie intentionnelle ordinaire (pour comprendre les énoncés faits dans un jeu de langage, un interprète doit s'appuyer sur la « façon commune d'agir » des êtres humains décrite en termes intentionnels); de l'autre, il n'y a pas une liste bien arrêtée de conditions $(c f . P U \mathbb{S} 183)$ les mêmes pour tous les locuteurs - telles que, si elles étaient réalisées, il ne pourrait pas ne pas y avoir compréhension : les conditions varient suivant les individus et les circonstances ( $c f$. aussi $P U \mathbb{S} 179$ et $B B$ p. 114 $)^{12}$.

12. Colin McGinn croit qu'il est correct de qualifier de «béhavioriste " le point de vue de Wittgenstein mais ajoute: "Je n'entends pas suggérer que Wittgenstein est un béhavioriste réductionniste au sens classique du terme ; je veux seulement dire qu'il est prêt à lier au comportement [d'un individu] l'attribution de concepts psychologiques indépendamment de tout état interne.Cela est tout à fait compatible avec la reconnaissance du fait [...] que la notion de comportement en cause implique [involves] essentiellement des notions psychologiques, c'est-à-dire doit être caractérisée en termes psychologiques, par exemple comme l'action intentionnelle d'une personne» (Wittgenstein on Meaning, Oxford, Blackwell, 1984, p. 34). 


\section{L'explication du sens}

Une condition d'un langage commun, semble-t-il, est non seulement que la signification soit objective mais que l'usage soit régi par des règles strictes. C'est apparemment ce qu'entendait Frege par le réquisit de la "détermination du sens " plus tard repris par Wittgenstein dans le Tractatus $(c f . T 3.23 \text { et } P U \mathbb{S} \mathbb{S} 71 \text { et } 99)^{13}$. Les langues naturelles cependant ne satisfont pas cette condition. Wittgenstein consacre une longue série de remarques à ce sujet et cite des exemples d'expressions dont l'usage, dit-il, n'est pas " partout circonscrit pas des règles » $(P U \mathbb{S} 68)$. Comment, si c'est le cas, la communication entre locuteurs est-elle possible ? La réponse du mentaliste - une réponse à laquelle souscrivaient peut-être Frege et Wittgenstein à l'époque de la rédaction du Tractatus - est que dans leur esprit ou, comme il dit ici, dans le "médium de la compréhension » $(P U \mathbb{S} 102)$, il n'y a rien d'indéterminé : pour le mentaliste, "quelqu'un qui prononce une phrase et la signifie ou la comprend opère ce faisant un calcul d'après des règles définies » $(P U \mathbb{S} 81)$. Mais dire cela revient à admettre soit que les règles sont contrairement aux apparences des règles strictes ou "définies " (ce que Wittgenstein nie dans les Recherches), soit qu'elles sont pour ainsi dire des règles de la pensée et non des règles du langage, ce qui n'aide pas à expliquer comment il peut y avoir une compréhension commune de ces expressions. La solution consiste pour Wittgenstein à reconnaître qu'un langage commun n'exige pas des règles de ce genre. Un autre problème va ensuite se poser : comment, si tel est le cas, une explication du sens est-elle possible ? Une question qui va nous ramener à l'un des thèmes de la précédente section.

Pour beaucoup d'expressions de la langue, il n'est pas toujours possible de fournir une réponse définie à la question "S'applique-t-elle ou non dans ce cas ? " L'exemple bien connu de Wittgenstein est celui du mot « jeu » un terme dit de " ressemblance de famille ». Il s'agit d'un terme de ressemblance de famille au sens suivant : les jeux ont plusieurs propriétés en commun, mais (a) il n'y en a aucun qui les possède toutes (une activité doit néanmoins en posséder plusieurs pour qu'elle soit correctement qualifiée de « jeu ») et (b) il n'existe pas (ou pas nécessairement) une propriété que tous ont en commun. Il pourrait y avoir par exemple une activité à laquelle les

La remarque me semble juste mais le qualificatif de "béhavioriste " peut à mon avis prêter à confusion. Au sens où l'emploie McGinn, un "béhavioriste » est simplement celui qui croit qu'on peut attribuer de façon justifiée des états psychologiques sans que les justifications fassent référence à des états internes de celui à qui on les attribue (des " états internes » entendus au sens d'une psychologie scientifique ou de la neurophysiologie). Pour une conception des états mentaux que l'on pourrait qualifier de " wittgensteinienne " en un sens large, $c f$. Lynne Ruder Baker, Explaining the Attitudes. A Practical Approach to the Mind (Cambridge : Cambridge University Press, 1995), en particulier chap. 6-8. (Baker ne note cependant pas les similitudes entre ses vues et celles de Wittgenstein).

13. Cf. sur ce point les remarques de N. Malcolm dans Wittgenstein : Nothing is Hidden (Oxford, Blackwell, 1986), pp. 36 et suiv. 
locuteurs refusent le qualificatif de " jeu " parce qu'elle ne possède pas un nombre "suffisant» des propriétés pertinentes ou qu'elle n'a aucune des propriétés tenues pour «centrales » ou plus typiques des jeux - le fait par exemple d'être divertissante (la règle d'usage du mot se formulerait en partie : " Pour qu'elle soit un jeu, une activité doit posséder un nombre suffisant des propriétés $\mathrm{A}, \mathrm{B}, \mathrm{C}, \ldots$ ou peut-être moins à condition qu'il s'agisse de propriétés plus typiques des jeux). Mais, pour une autre activité, il pourrait ne pas y avoir de réponse précise à la question « Le terme s'applique-til ou non dans ce cas ? " : les locuteurs ne s'accordent peut-être pas pour dire si le nombre de ses propriétés pertinentes est "suffisant » ou si l'une d'elles doit être considérée comme plus typique des jeux ${ }^{14}$. L'idée suivant laquelle il y aurait " caché » dans leur esprit des règles d'usage strictes pour l'usage du mot ne paraît pas très plausible.

Des règles strictes, dit Wittgenstein, ne sont de toute façon pas nécessaires. Il suggère (entre autres) une analogie entre l'usage d'un mot et la pratique d'un sport : on n'a pas plus de raison de penser que l'usage du mot " jeu » est " partout circonscrit par des règles » (qu'il obéit à des règles strictes) qu'on en a de le penser, par exemple, de la pratique du tennis - ce qui ne nous empêche pas d'en faire usage (pas plus que cela ne nous empêche de jouer au tennis) (cf. $P U \mathbb{S} 68$ ).

Mais un problème se pose en ce qui concerne l'explication de l'usage de ces expressions. Pour expliquer l'emploi d'une expression régi par des règles strictes, il suffit d'énoncer les règles et d'indiquer la façon dont on les applique. Comment procède-t-on pour un terme tel que " jeu »? Wittgenstein répond : l'explication va consister " [à décrire] des exemples de différentes espèces de jeux, [à montrer] comment toute une variété d'autres jeux peuvent être construits par analogie avec ceux-ci, [à dire] qu'[on inclurait] difficilement [telle ou telle activité] parmi les jeux, etc. » $(P U \mathbb{S} 75)$ Ainsi, " on donne des exemples et on veut que ceux-ci soient compris en un sens particulier ». $(P U \mathbb{S} 71)$ Mais il ajoute : " par cette dernière expression je ne veux pas dire que [l'apprenti] doit voir dans ces exemples quelque chose de commun que, pour une raison ou une autre, je n'étais pas en mesure d'exprimer ; mais plutôt qu'il doit employer les exemples d'une façon déterminée " (Ibid.). Le mentaliste pourrait en effet objecter qu'une explication qui se base sur des exemples n'est (comme le lui fait dire Wittgenstein) qu'un « moyen indirect d'explication " (Ibid.) : le maître doit avoir à l'esprit une « définition non formulée » du mot (qui indique ce qu'ont en commun tous les jeux ou qui

14. Un autre exemple différent de celui des termes de ressemblance de famille est celui des noms propres ( $c f . P U \$ 79)$. Si une description représente la règle d'emploi du nom « $\mathrm{N}$ », il peut ne pas y avoir accord sur la réponse à donner à la question « N existe-t-il ? " (par exemple «Moïse a-t-il existé ? ») parce que la règle (la description) ne spécifie pas les propriétés tenues pour « essentielles» (ou au contraire « accessoires ») quand il s'agit de décider de l'existence ou non-existence de N (quelles propriétés considérer comme "essentielles " si on veut déterminer si Moïse a réellement existé ?). 
fournit au moins une règle d'usage " définie " du mot) et son but en donnant les exemples est de produire la même définition dans l'esprit de l'apprenti.

On a vu que la compréhension d'un mot consiste dans la disposition à agir en l'employant. La réponse au mentaliste revient en conséquence à suggérer une autre description du processus d'apprentissage : l'usage du mot est enseigné au moyen d'exemples assortis des explications usuelles; lorsque l'apprenti a " compris ", il se met à l'employer comme le maître (et tous les autres locuteurs) et le but a été atteint. On n'a pas à postuler une étape intermédiaire - quelque chose tel que la " fixation » d'une définition ou d'une règle dans l'esprit de l'apprenti - comme condition nécessaire de la compréhension. Bref, on peut concéder peut-être au mentaliste que ces explications ne sont effectivement pas les " meilleures" $(P U \mathbb{S} 71)$ mais, pour un mot tel que « jeu », on n'en a pas d'autres et celles-ci remplissent de toute façon parfaitement leur rôle (comme dit Wittgenstein, «c'est ainsi que nous jouons le jeu » $[P U \$ 71])$.

Un autre problème se pose néanmoins en ce qui concerne cette fois l'explication de toutes les expressions de la langue (et non seulement l'explication d'un terme de ressemblance de famille). Wittgenstein remet ici en question la distinction qu'il avait apparemment acceptée un peu auparavant

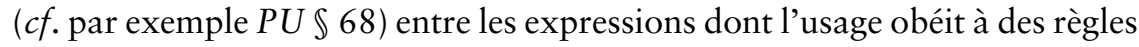
strictes et les autres expressions :

J'ai dit de l'usage d'un mot [par exemple du mot « jeu »] qu'il n'est pas partout circonscrit pas des règles. Mais à quoi donc ressemble un jeu [entre autres un jeu de langage] qui soit partout circonscrit par des règles ? Dont les règles ne laissent jamais se manifester un doute, [mais] qui comblent [d'avance] tous les trous [où un doute peut surgir] ? - Ne pouvons-nous pas penser à une règle qui régit l'application de la règle et un doute que celle-ci lève - et ainsi de suite? $(P U \mathbb{8} 84)$

Pour prendre un de ses exemples, supposons que l'on ait comme règle d'emploi des noms de couleurs un tableau mettant en corrélation des mots et des échantillons de couleur (les mots forment sur le tableau une première colonne à gauche et les échantillons une seconde à droite). Quelqu'un qui a des « doutes " sur son application de la règle (que représente le tableau) pourrait se demander quelle méthode de lecture du tableau adopter : celle que la plupart d'entre nous considérons comme la méthode "normale " ou peut-être une autre méthode. La méthode dite "normale» est la suivante : le premier mot de la colonne de gauche est le nom de la couleur dont l'échantillon est le premier de la colonne de droite, le deuxième le nom de la couleur dont l'échantillon est le deuxième de la colonne de droite, etc. Une autre méthode (que l'on qualifierait peut-être de "non-standard») pourrait être celle-ci (je cite encore ici l'exemple de Wittgenstein) : le premier mot de la colonne de gauche serait le nom de la couleur dont l'échantillon est le deuxième de la colonne de droite, le deuxième le nom de la couleur dont l'échantillon est le troisième de la colonne de droite... et le dernier le nom de 
la couleur dont l'échantillon est le premier de la colonne de droite (cf. PU $\mathbb{S}$ 86). Chaque méthode constitue une règle pour l'application de la première (le tableau) - on peut sans difficulté imaginer d'autres règles - et si quelqu'un a aussi des doutes sur l'application de l'une d'elles, on peut facilement, dit Wittgenstein, se représenter encore " d'autres règles pour $\left[l^{\prime}\right]$ expliquer» (Ibid.), etc ${ }^{15}$. Le problème est que les explications vont toujours dans ce cas rester « incomplètes »; comme le dit l'interlocuteur (avec une pointe d'impatience !) : " Mais comment une explication peut-elle bien m'aider à comprendre si elle n'est pas la dernière ? L'explication n'est alors jamais terminée; je ne comprends donc toujours pas et je ne comprendrai jamais ce que veut dire [meint] [celui qui l'enseigne] » (PU $\$ 87)$.

Le préjugé mentaliste transparaît dans sa remarque : le maître a toujours quelque chose de bien défini à l'esprit mais, puisqu'il n'y a pas d'explication " complète » d'une règle, il ne pourra jamais le communiquer à l'apprenti sauf peut-être "par hasard » - si l'apprenti arrive à deviner ce que «veut dire » le maître. À cela Wittgenstein répond : "Comme si une explication était pour ainsi dire suspendue en l'air si elle n'était pas étayée par une autre " (Ibid.). L'explication serait "incomplète " si elle ne répondait pas à tous les doutes exprimés par l'apprenti; mais elle ne peut traiter de tous les doutes concevables car le processus d'explication serait sans fin : " [o]n pourrait dire : une explication sert à écarter un malentendu [Missverständnis : malentendu ou mécompréhension] ou à le prévenir c'est-à-dire un malentendu qui pourrait survenir sans cette explication mais non tous les malentendus que je peux m'imaginer » (Ibid.). Il est juste en un sens de dire qu'une explication n'est jamais la "dernière " (puisqu'il n'y a pas de terme aux questions qu'on peut poser sur le sens d'une expression ou sur l'application d'une règle) mais en pratique les explications prennent toujours fin quelque part - quand l'apprenti comprend, c'est-à-dire lorsqu'il se met à employer le mot comme le comprend le maitre (et le comprennent les autres locuteurs).

Wittgenstein ferait sans doute une remarque similaire au sujet de l'apprentissage de l'usage du mot « cube » à l'aide d'un dessin (cf. section 2) : comme on peut associer au dessin plus d'une « méthode de projection » et associer à son tour à une méthode donnée plusieurs applications possibles,

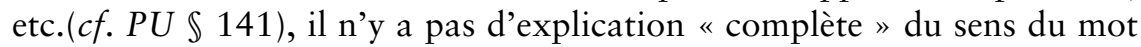
« cube » à l'aide d'un dessin (ni de tout autre moyen d'explication, comme

15. Pour prendre un autre exemple, si quelqu'un ignore (ou a des doutes sur) la signification de "Moïse ", on peut expliquer sa signification en donnant une description, par exemple : "Par le nom de "Moïse " je comprends l'homme, s'il y a eu un tel homme, qui a conduit les Israélites hors d'Égypte, peu importe le nom qu'il portait et peu importe ce qu'il peut avoir fait par ailleurs» $(P U \mathbb{\$} 87)$. Mais « des doutes semblables à ceux au sujet du nom "Moïse » sont possibles au sujet des mots qui figurent dans cette explication " (Ibid.) : quel pays était appelé l' "Égypte », qui étaient les «Israélites », etc. — des questions qui, dit Wittgenstein, «ne prennent fin [nulle part]. » 
il dirait sans doute) ; cependant on peut enseigner son usage au moyen du dessin et, dans la plupart des cas, l'explication atteint son but parce que les êtres humains tendent généralement à faire du dessin une même application. Wittgenstein ne nous dit pas pourquoi c'est le cas mais il semble que la raison soit que les êtres humains participent d'une nature commune et partagent un arrière-plan culturel et des "formes de vie " (peut-être propres à certains groupes linguistiques) tels qu'ils vont considérer comme «normales» ou " naturelles » certaines façons d'appliquer les règles et non d'autres ( $c f$. aussi $P U \rrbracket 241)$.

Comme je le montre dans ce qui suit, cette " solution » du problème du caractère incomplet des explications préfigure la "solution» du "paradoxe " au sujet des règles dont il est plus loin question dans les Recherches.

\section{Un « paradoxe » au sujet des règles}

Plusieurs passages des Recherches cités dans les deux précédentes sections laissent penser que Wittgenstein souscrit à ce qu'on pourrait appeler un " holisme des règles " (apparenté au " holisme des croyances " ou " holisme des concepts » dont parlent les philosophes aujourd'hui). La règle d'usage (partielle) du mot " cube " fournie par le dessin d'un cube ne " détermine ", semble-t-il, une application que si elle est accompagnée d'une «méthode de projection » et, puisqu'on peut imaginer « différentes applications » de cette méthode, celle-ci doit être assortie de directives pour sa propre application et celles-ci encore d'autres règles, etc. (cf. section 2). Il semble, pour prendre un autre exemple que j'ai cité, qu'un tableau d'échantillons ne représente une règle d'usage des noms de couleurs que si on lui a adjoint une "méthode de lecture ", laquelle présuppose à son tour d'autres instructions pour son application et celles-ci également d'autres règles et ainsi de suite. Comme on vient de voir, un problème que pose le holisme est celui du caractère incomplet des explications (comment expliquer à quelqu'un une règle si elle présuppose un nombre indéfini d'autres règles ?). Un autre problème est celui de savoir ce que l'on pourra appeler " une application correcte " : s'il existe toujours plus d'une façon de comprendre (auffassen) ou d'interpréter (deuten) une règle, comment peut-elle dicter telle application plutôt que telle autre $^{16}$ ?

Wittgenstein parle à ce propos d'un " paradoxe ": " Notre paradoxe était le suivant : une règle ne pourrait déterminer aucune conduite parce que

16. Pour un exposé plus détaillé de mon interprétation de ces textes, voir « La source du paradoxe de Wittgenstein à propos des règles » (Canadian Journal of Philosophy, 28, 1998), un article dans lequel je compare entre autres mon interprétation à celle de $\mathrm{S}$. Kripke dans Wittgenstein on Rules and Private Language. 
n'importe quelle conduite pourrait être mise en accord avec la règle [...]. De la sorte, il n'y aurait ni accord ni conflit [entre une action et ce qu'elle

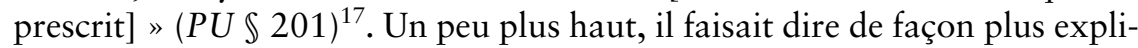
cite à son interlocuteur : « Mais comment une règle peut-elle m'apprendre ce que je dois faire en $c e$ point ? Peu importe ce que je fais, cela pourra s'accorder avec la règle suivant une interprétation ou une autre [suivant une manière ou une autre de la comprendre] » $(P U \mathbb{S} 198)$. En quoi par exemple consiste une application correcte de la règle que représente le tableau d'échantillons de couleur si n'importe quelle application peut s'accorder avec le tableau suivant un mode de lecture ou un autre ? On pourrait être tenté de dire, comme semble-t-il ici l'interlocuteur, qu'une fois choisie l'interprétation suivant laquelle on veut la suivre, une application de la règle et une seule sera dorénavant correcte : "Il me semble [dit l'interlocuteur] que j'aie donné une interprétation de la règle avant de la suivre et que cette interprétation suffise à déterminer ce que je dois faire pour la suivre dans un cas particulier. Si je comprends [auffasse] la règle comme je l'ai comprise, il ne lui correspond que cette action " (BGM VI $\$ 30)$. À quoi Wittgenstein répond : "Toute interprétation, de même que ce qu'elle interprète, est suspendue en l'air ; elle ne peut lui procurer aucun appui. Les interprétations ne déterminent pas en

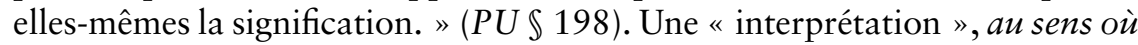
l'entend l'interlocuteur, serait "suspendue en l'air " puisqu'elle requerrait elle-même une interprétation qui fixe son contenu, ce qui entraîne une régression indéfinie : une "interprétation » (ou peut-être le fait d'avoir présente à l'esprit une telle interprétation) ne suffit pas déterminer le contenu d'une règle (à dire en quoi consiste son application correcte) dans la mesure où, semble-t-il, elle exige qu'elle soit interprétée.

On peut imaginer la réplique suivante de l'interlocuteur : " Il n'est peut-être pas nécessaire à chaque fois que je suis une règle de choisir ou d'avoir présente à l'esprit l'interprétation sous laquelle je veux la suivre, mais le holisme implique qu'il doit y avoir une telle interprétation (laquelle peut se formuler par une autre règle) de sorte que je dois au moins la connaître (elle doit se trouver en quelque manière dans mon esprit) et connaitre toutes les autres règles ou interprétations qui se tiennent derrière celle-là (ce qu'on

17. Bon nombre d'interprètes des Recherches soutiennent qu'il n'y a pas ici pour Wittgenstein d'authentique "paradoxe. " Il parle pourtant de "notre paradoxe ». De plus, comme l'atteste un manuscrit non publié, il avait d'abord écrit (je souligne) : "J'étais (plus tôt) dans la difficulté qu'une règle ne pourrait déterminer aucune conduite parce que n'importe quelle conduite pourrait être mise en accord avec la règle » au lieu de (comme dans le texte publié des Recherches) : "Notre paradoxe était le suivant : une règle ne pourrait déterminer aucune conduite parce que n'importe quelle conduite... ». Il y a donc une "difficulté » qui requiert une solution (Kripke a raison sur ce point). Le manuscrit en question est cité par G. P. Baker et P. M. S. Hacker, Wittgenstein : Rules, Grammar and Necessity (Oxford : Blackwell, 1985). p. 147. Hacker et Baker partagent (assez curieusement) l'interprétation courante selon laquelle il n'y a pas ici pour Wittgenstein de véritable paradoxe (cf.ibid. pp. 132 et suiv.). 
pourrait appeler l'ensemble des " présupposés » de la règle), sinon je ne peux agir en accord avec ce qu'elle demande ». Dans un de ses cours, Wittgenstein répond comme suit :

Lorsqu'une personne [n'agit] qu'en suivant le tableau [mettant par exemple en corrélation des noms et des échantillons de couleur] sans qu'on lui ait donné [la première des deux méthodes de lecture], connaissait-elle cette dernière règle [la première méthode] ? On pourrait soutenir que si elle ne l'avait pas connue, elle n'aurait pu utiliser le tableau comme elle l'a fait. Cela peut créer l'impression que la chaîne des raisons [des interprétations ou justifications] n'a pas de fin [et] que seules les raisons écrites ont une fin. Mais doit-on connaître cette règle [la première méthode] quand on emploie le tableau ? Non, on [agit] simplement [en accord avec le tableau]. À la question " Pourquoi, après qu'on lui a appris à utiliser le tableau, s'est-elle mise à agir [en le suivant] ? » la réponse est simplement qu'elle l'a fait à moins qu'on veuille citer une autre règle [pour justifier sa façon de le suivre]. On pourrait objecter que [si tel est le cas], une personne agit comme un automate, sans comprendre. Mais, souvent, en comprenant quelque chose on le fait simplement (WLC p. 132-133).

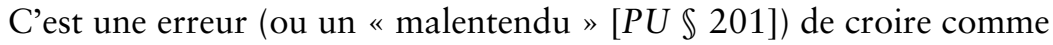
l'interlocuteur que le holisme implique que, avant de suivre une règle, on doit avoir présente à l'esprit l'interprétation sous laquelle on s'apprête à la suivre. Wittgenstein écrit dans les Fiches $(\$ 234)$ : «Ce n’est pas que ce symbole ne puisse plus être interprété, mais : je n'interprète pas. Je n'interprète pas parce que je me sens à l'aise [dans cette façon de le comprendre] ». Et au paragraphe suivant : " [J'emploie un tableau de l'horaire des chemins de fer] sans me préoccuper du fait qu'un tableau est susceptible de toutes sortes d'interprétations ». C'est par ailleurs également une erreur de croire que le holisme implique que l'on doit être apte à énoncer des interprétations ou justifications à l'appui de la façon dont on la suit (un enfant peut ne pas avoir cette aptitude). Comme l'affirme Wittgenstein de façon peut-être plus surprenante dans le texte du cours, on n'a pas même besoin pour pouvoir l'appliquer de connaître - au sens d'une connaissance propositionnelle l'interprétation sous laquelle on la suit et tout l'arrière-plan des autres règles qu'elle présuppose car elles sont en nombre infini et il n'est pas plausible postuler chez les locuteurs une semblable connaissance.

En quoi dès lors va consister la compréhension (ou connaissance) de la règle ? Dans le passage des Recherches qui traite du " paradoxe ", Wittgenstein fait cette remarque : " [...] il y a une compréhension [Auffassung] d'une règle qui $n$ 'est pas une interprétation mais qui se manifeste [...] par ce que nous appelons "suivre la règle " et "aller à son encontre" " $(P U \mathbb{S} 201)$. Comme il le dit également dans le texte du cours, comprendre la règle peut simplement consister à agir en la suivant. Les deux passages font écho à des remarques antérieures des Recherches - j'en ai cité quelques unes - lorsque Wittgenstein disait par exemple que " [l']application demeure un critère de

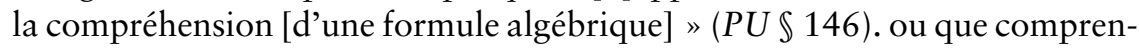


dre une règle consiste en une capacité pratique (cf.PU $\mathbb{2 0 2}$ : « suivre une règle est une pratique $»$ ).

L'interlocuteur répondrait maintenant peut-être ceci : " Assurément, une condition de l'existence d'un langage commun est que les locuteurs s'entendent sur la signification des mots qu'ils emploient. Ils doivent par conséquent partager les mêmes règles et tout l'arrière-plan de présupposés qui se tient derrière celles-là ; ils doivent connaître ces présupposés, sinon on ne voit pas comment il peut y avoir communication ». On peut déduire la réponse de Wittgenstein d'un passage des Recherches (PU $\mathbb{S} 239-242)$ mais je citerai un passage plus explicite des Remarques sur les fondements des mathématiques, qui se lit comme suit :

Il est vrai [comme dit l'interlocuteur] que tout peut d'une certaine façon se justifier. Mais le phénomène du langage [c'est-à-dire d'un langage partagé] repose sur la régularité, sur l'accord dans l'action.

Il est ici de la plus grande importance que nous tous ou l'immense majorité d'entre nous nous accordons sur certaines choses. Je peux par exemple être tout à fait certain que la plupart des gens qui voient cet objet vont le qualifier de vert. $[\ldots]$

Nous disons que, pour que les gens se comprennent entre eux, ils doivent s'accorder sur la signification des mots. Cependant le critère de cet accord n'est pas seulement un accord sur les définitions, par exemple les définitions ostensives - mais aussi un accord sur les jugements. Il est essentiel pour qu'il $\mathrm{y}$ ait compréhension que nous nous accordions sur un grand nombre de jugements (BGM VI $\mathbb{3} 39)$.

Il est correct de prétendre comme l'interlocuteur que n'importe quelle action peut être mise en accord avec une règle suivant une interprétation ou une autre ("tout peut d'une certaine façon se justifier») — c'est le «paradoxe » dont il était question plus haut ; mais c'est un fait que, à part quelques exceptions, tous les êtres humains s'accordent sur la façon d'appliquer les règles de la langue. Leurs comportements linguistiques exhibent beaucoup de "régularité ", d' " accord dans l'action ", quand ils décrivent par exemple les couleurs des objets. Comme disait Wittgenstein, on peut imaginer toutes sortes d'applications du dessin d'un cube considéré comme une règle d'usage de "cube ", mais il y a une application que la plupart jugent « normale » ou "naturelle » $(P U \$ 141)$. Par ailleurs, notre critère de l'accord quant à la signification ne consiste pas seulement en un accord sur les règles d'usage et leur formulation (une définition ostensive, un tableau d'échantillons pour l'emploi des noms de couleurs, etc.), mais en un accord sur l'application (consistant à émettre par exemple les mêmes jugements sur les couleurs des objets). L'accord sur la formulation ne suffit pas parce que, comme le rappelle l'interlocuteur, il y a toutes sortes de façons de les suivre (de les comprendre ou "interpréter ») et c'est précisément la façon de les appliquer dans des jugements qui montre comment elles sont comprises. Il 
est en un sens exact de dire que tous les locuteurs doivent avoir une même compréhension et accepter les mêmes interprétations des règles du langage (c'est en effet une condition d'un langage commun) — non cependant au sens où le croit l'interlocuteur : d'une part, il n'est pas nécessaire d' « interpréter » les règles (ou d'avoir à l'esprit les interprétations sous lesquelles on veut les suivre) ; de l'autre, la compréhension commune particulière qu'en ont les locuteurs se manifeste dans l'application qu'ils en font. Bref, contrairement à ce que voudrait dire l'interlocuteur, on doit tenir pour une condition d'un langage partagé l' "accord dans l'action", non l'accord dans les " interprétations ». Ni l'accord dans les "interprétations », ni la possession d'une connaissance propositionnelle (implicite) commune des présupposés des règles n'est une condition d'un langage commun ${ }^{18,19}$.

Reste la question soulevée au départ par l'interlocuteur : si on peut donner d'une règle toutes sortes d'interprétations, comment peut-il y avoir accord ou conflit — " collision ", disait Wittgenstein (cf. section 2) - avec ce qu'elle prescrit ? La réponse est implicite dans les passages déjà cités. Comme le disait Wittgenstein, ce n'est pas n'importe quelle action (n'importe quel jugement ou application) qui peut s'accorder avec l'image d'un cube considérée comme règle d'emploi de " cube »; il peut y avoir « collision » entre l'image et son application «dans la mesure où l'image nous fait nous attendre à un [certain] usage [aller chercher par exemple un cube quand on nous en donne l'ordre], car les gens font en général de cette image cette application » $(P U \mathbb{S} 141)$. La plupart des gens ont tendance à

18. On lit dans les Remarques sur les fondements des mathématiques: "Comment le mot " Dalle " [ou l'ordre " Apporte une dalle "] peut-il me montrer ce que je dois faire si après tout je peux mettre en accord n'importe quelle action avec n'importe quelle interprétation ? Comment puis-je suivre une règle si, peu importe comment j'agis, cela peut être interprété comme la suivre ? Que dois-je savoir pour obéir à l'ordre ? Y a-t-il un savoir dont il résulte que la règle ne peut être suivie que de cette façon ? » On trouve bien sûr ici une nouvelle formulation du paradoxe. Wittgenstein répond : "Je dois parfois savoir quelque chose, je dois parfois interpréter la règle avant de l'appliquer. [...] [Mais] interpréter a une fin » (BGM VI $\$ 38$ ).

19. On pourrait objecter à Wittgenstein que si un locuteur était apte à rendre explicites certains présupposés d'une règle de la langue (à dire par exemple en quoi consiste la méthode de lecture usuelle du tableau d'échantillons de couleur) et qu'un autre locuteur (disons un enfant) n'avait pas cette aptitude tout en ayant les mêmes compétences linguistiques que le premier (dans son usage, entre autres, des noms de couleurs), on serait enclin à dire que le second locuteur connaît implicitement des présupposés de la règle que le premier connaît explicitement. $\mathrm{Si}$, autrement dit, le comportement linguistique du second montre qu'il accepte des interprétations de la règle qu'accepte le premier alors que celui-ci a de surcroît l'aptitude à les énoncer explicitement, il semble aussi sensé d'attribuer la connaissance de ces interprétations au second locuteur qu'au premier. Wittgenstein répondrait (je suppose) que le critère pour dire que le premier connaît des présupposés de la règle est qu'il les a effectivement énoncés mais que ce critère n'est pas satisfait chez le second : ce dernier a la même compréhension de la règle que l'autre (il " connaît » par exemple la méthode de lecture courante du tableau) seulement au sens où cette compréhension se manifeste dans son comportement (dans l'application qu'il fait du tableau) -- peu importe ce qu'il peut avoir dans l'esprit ou le cerveau. 
faire une même application du dessin d'un cube après avoir reçu les instructions habituelles et c'est en ce sens seulement qu'il peut y avoir conflit (ou accord) entre la règle et son application : l' " accord dans l'action » (la convergence des jugements) ou l' « usage constant » qu'en font ses utilisateurs (cf.PU $\mathbb{S} \mathbb{1 9 0}$ et 198) est ce qui définit son application correcte. Il n'y a pas à proprement parler de compréhension " correcte " d'une règle si ce n'est celle que la plupart de ses utilisateurs jugent « correcte» (cf. WLFM p. 183$)^{20}$.

On peut voir la similitude entre la solution du problème du caractère incomplet des explications et celui de savoir comment est possible un accord (ou un conflit) entre ce que prescrit une règle et son application. La " solution » dans le premier cas consistait à reconnaître que des explications supplémentaires ne sont pas en fait nécessaires (elles prennent toujours fin quelque part) dans la mesure où la plupart des locuteurs, après avoir subi un apprentissage semblable, tendent à faire la même application (par conséquent à avoir la même compréhension) des règles du langage. La "solution » du second problème est similaire : c'est un trait contingent de la nature humaine que les locuteurs soient disposés à faire la même application des règles du langage - l'application qu'ils vont considérer comme " correcte "; ils se sentent " à l'aise ", comme dit Wittgenstein, dans une certaine manière - en fait commune - de les comprendre.

20. Pour certains commentateurs, Wittgenstein soutient que l'idée d'application correcte n'a de sens que si l'on parle de règles communes, ce qui entraîne le caractère nécessairement social de tout langage. S. Kripke par exemple écrit : ce à quoi s'oppose l'argument de Wittgenstein contre la possibilité d'un langage privé « est ce que l'on pourrait appeler le “ modèle privé " de ce qu'est suivre une règle [c'est-à-dire le point de vue selon lequel] la notion que quelqu'un suit une règle donnée pourrait être analysée simplement en termes de faits relatifs à celui qui la suit et seulement à lui, sans référence à l'appartenance à une communauté plus large [d'utilisateurs]». (Wittgenstein on Rules and Private Language, p. 109). N. Malcolm a soutenu une interprétation (en partie) similaire (dans "Wittgenstein on Language and Rules ", Philosophy, 64, 1989, pp. 5-28). G. P. Baker et P. M. S. Hacker défendent l'interprétation opposée ("Malcolm on Language and Rules ", Philosophy, 65, 1990, pp.167-179). Wittgenstein ne rejette pas selon eux la possibilité d'un idiolecte à la condition que celui-ci ne soit pas "privé » (est privé un langage qui ne peut logiquement être compris que par celui qui le parle). Ils écrivent, je pense avec raison : « Nous prétendons que Wittgenstein a soutenu, et soutenu à bon droit, que l'accord dans les jugements et les définitions, le consensus au sein de la communauté [des locuteurs] sur [...] ce qu'est appliquer correctement une règle sont présupposés par un langage partagé, des concepts communs et des règles en usage dans une communauté. Mais la possession de concepts, le fait de suivre des règles et la maîtrise d'un langage présupposent non pas que ceux-ci soient partagés mais qu'ils puissent l'être [...] ». (p. 171). On peut en effet montrer (je ne le ferai pas ici) qu'il découle de certains passages des Recherches qu'un idiolecte non privé est possible en autant qu'un tiers puisse l'interpréter (cf. $P U \mathbb{S}$ 206-207). 


\section{Conclusion : la méthodologie des Recherches}

Pour terminer, je pense que ce qui précède éclaire quelques-unes des remarques métaphilosophiques (ou méthodologiques) des Recherches. Le philosophe, dit Wittgenstein, s'efforce de déterminer l'« essence du langage, des

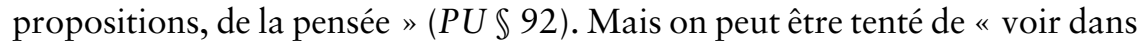
l'essence [du langage ou de la pensée] non quelque chose qui est déjà étalé sous nos yeux [...] mais quelque chose qui gît sous la surface [ou] à l'intérieur [...] » (Ibid.). On lit également plus loin : «La philosophie met simplement tout devant nous et n'explique ni ne déduit rien. - Puisque tout est étalé sous nos yeux, il n'y a rien à expliquer. Car ce qui peut-être est caché ne nous intéresse pas» $(P U \mathbb{S 1 2 6 )}$.

Pour savoir en consiste l'essence du langage, comme il dit également ici, on doit tenir compte de sa "fonction " et de sa « structure " $(P U \mathbb{S} 92)$. On a vu en effet (section 1) que la signification d'une expression dépend de sa "fonction ", c'est-à-dire de son " rôle " ou son " usage " dans des jeux de langage ${ }^{21}$. Pour prendre l'exemple du « jeu du constructeur» du début des Recherches, quand on a décrit les actions des participants et les circonstances des actions (donc le rôle des signes dans le jeu), on a tout dit sur leur signification. Il est clair en particulier dans le cas d'un jeu de langage "simple " (comme le jeu du constructeur) que tout en ce qui concerne la signification des signes est publiquement accessible aux locuteurs (ainsi qu'aux interprètes du langage et à ceux qui en font l'apprentissage). Rien ici n'est " caché ».

Pour le mentaliste, l'« important " dans le phénomène du langage est plutôt quelque chose qui se tient caché "sous la surface " ou "à l'intérieur ». J'ai cité plusieurs expressions de ce point de vue : (a) La communication consiste en une transmission de " pensées " (ce qui importe à un locuteur est de faire savoir à son auditeur ce qu'il a "en tête »). (b) Comprendre ou interpréter de telle ou telle façon une définition ostensive " désigne un processus [dans nos esprits] accompagnant l'action de prononcer et d'entendre la définition » $(P U \mathbb{S} 34)$. (c) Notre usage du langage obéit à des règles strictes, des règles situées "à l'arrière-plan - cachées dans le médium de la compréhension » $(P U \mathbb{S} 102)$. (d) Une explication du sens demeure toujours "incomplète "; l'apprenti ne peut que " deviner » ce que le maître veut lui faire comprendre (et ce qu'il veut lui faire comprendre est quelque chose de parfaitement déterminé au moins dans l'esprit du maître) (cf.PU $\mathbb{S} 210)$. (e) La compréhension d'une règle consiste en une « interprétation » (quelque chose qui a lieu dans notre esprit). ( $f$ ) L'accord sur la signification suppose une même compréhension des règles du langage, une conception partagée de leurs présupposés sous la forme d'une connaissance tacite.

21. Il ne dit cependant pas ici ce qu'il faut comprendre par l'expression «structure du langage ». 
Le mentaliste veut ainsi expliquer le phénomène d'un langage commun - expliquer comment est possible la communication, en quoi consiste la compréhension, comment peut s'instaurer un accord sur la signification en postulant des pensées (des « sens » entendus à la façon de Frege, etc.), des processus internes (de " compréhension» ou d' "interprétation»), des "définitions non formulées ", une connaissance commune implicite des présupposés des règles du langage, etc ${ }^{22}$.

«Puisque tout est étalé sous nos yeux, il n'y a rien à expliquer". L' « important » se trouve "à la surface » de nos pratiques linguistiques; il n'est pas nécessaire d'aller au-delà, à " l'intérieur ", pour connaître l'essence du langage. On n'a pas à la découvrir, il suffit de la décrire.

22. Que veut dire Wittgenstein lorsqu'il écrit : « Car ce qui peut-être est caché ne nous intéresse pas » $(P U \mathbb{1 2 6})$ ? Peut-être fait-il allusion à une explication qui fait appel à l'idée d'un mécanisme neurophysiologique. On pourrait par exemple expliquer par un tel mécanisme le fait que celui à qui on vient d'enseigner une règle se mette à la suivre correctement, c'est-à-dire de la manière dont on le voulait : le processus d'apprentissage, comme il écrit dans les Fiches, pourrait avoir pour effet de créer dans le cerveau de l'apprenti « la machine correcte qui, une fois choisie, va le pousser automatiquement [à suivre la règle comme on veut qu'il la suive] » $(Z$ $\$ 304)$. Mais Wittgenstein ajoute : "Il pourrait très bien se faire que quelque chose du genre se produise dans notre cerveau, mais cela ne nous intéresse pas " (Ibid.). Pourquoi ? Peut-être parce que même si on découvrait chez un individu un tel mécanisme, il est parfaitement imaginable que le mécanisme soit différent chez un autre mais que le critère usuel pour décider si la règle est appliquée correctement soit satisfait chez les deux ( $c f . P U \$ 376)$. On n’aurait pas dans ce cas réussi à « expliquer » ce qu'est " suivre correctement la règle » (c'est-à-dire la suivre comme nous tous). 